\title{
Henoch-Schönlein purpura: recurrence and chronicity
}

\author{
Camila S. Alfredo, ${ }^{1}$ Nicole A. Nunes, ${ }^{2}$ Claudio A. Len, ${ }^{3}$ Cássia M. P. Barbosa, ${ }^{4}$ \\ Maria Teresa R. A. Terreri, 5 Maria Odete E. Hilário6
}

\begin{abstract}
Objectives: To describe a group of patients treated at our service for Henoch-Schönlein purpura, with emphasis on recurrent and chronic cases, and to compare clinical and demographic characteristics of patients with monocyclic and recurrent disease.

Methods: Data on 67 patients who had been treated since disease onset were analyzed. Twelve patients were excluded because they failed to return for follow-up consultations after less than 3 months, leaving a total of 55 children in the study sample. Recurrence was defined as the presence of a fresh episode after a period of at least 3 months without symptoms, and cases were defined as chronic when cutaneous, abdominal and renal manifestations persisted for a period of 12 months or more.
\end{abstract}

Results: Recurrence was observed in $8 / 55$ patients (14.4\%) and four cases were chronic (7.2\%). In 29/55 patients (52.7\%), infection was identified as the trigger factor. A monocyclic clinical course was observed in 43 patients ( 26 of whom were girls, with a mean age of 5.4 years). Gastrointestinal and renal involvement was observed in 55.8 and $20.9 \%$ of patients, respectively. Among the 12 patients with recurrent or chronic Henoch-Schönlein purpura, three had arthritis, four exhibited signs and symptoms of abdominal involvement and seven of kidney disease: microscopic hematuria in five, macroscopic hematuria in one and hematuria with proteinuria in one other. Late onset was the only variable related to recurrence $(p<0.05)$.

Conclusions: As is observed in medical literature, monocyclic cases are more common among children with early onset disease. Patients with Henoch-Schönlein purpura should be followed over the long term, since recurrent and chronic cases account for more than $20 \%$ of the total.

J Pediatr (Rio J). 2007;83(2):177-180: Henoch-Schönlein purpura, purpura, nephritis, vasculitis.

\section{Introduction}

Henoch-Schönlein purpura (HSP) is the most common type of systemic vasculitis in childhood and is characterized by palpable, non-thrombocytopenic purpura, sometimes accompanied by arthritis and/or arthralgia, abdominal pains, hemorrhage gastrointestinal and/or nephritis.

Although its cause is unknown, it is known that immunoglobulin $A(\operatorname{IgA})$ plays an important role in its

1. Médica pediatra. Especializanda, Setor de Reumatologia, Disciplina de Alergia, Imunologia e Reumatologia, Departamento de Pediatria, Universidade Federal de São Paulo - Escola Paulista de Medicina (UNIFESP-EPM), São Paulo, SP, Brasil.

2. Médica pediatra. Especialista em Reumatologia Pediátrica, Disciplina de Alergia, Imunologia e Reumatologia, Departamento de Pediatria, UNIFESP-EPM, São Paulo, SP, Brasil.

3. Professor adjunto, Disciplina de Alergia, Imunologia e Reumatologia, Departamento de Pediatria, UNIFESP-EPM, São Paulo, SP, Brasil.

4. Mestre. Professor assistente, Disciplina de Alergia, Imunologia e Reumatologia, Departamento de Pediatria, UNIFESP-EPM, São Paulo, SP, Brasil.

5. Professora afiliada, Disciplina de Alergia, Imunologia e Reumatologia, Departamento de Pediatria, UNIFESP-EPM, São Paulo, SP, Brasil.

6. Professora associada, Disciplina de Alergia, Imunologia e Reumatologia, Departamento de Pediatria, UNIFESP-EPM, São Paulo, SP, Brasil.

Manuscript received Sep 01 2006, accepted for publication Nov 292006.

Suggested citation: Alfredo CS, Nunes NA, Len CA, Barbosa CM, Terreri MT, Hilário MO. Henoch-Schönlein purpura: recurrence and chronicity. J Pediatr (Rio J). 2007;83(2):177-180.

doi 10.2223/JPED.1595 
immunopathogenesis. In up to $50 \%$ of cases, an upper respiratory infection (URI) precedes the disease by days or weeks. ${ }^{1,2}$ It has been suggested that group A beta-hemolytic streptococcus may be a trigger factor, as may the use of medicines and vaccines and contact with specific food allergens, such as colorings and preservatives. ${ }^{1,2}$

Approximately one third of patients have at least one recurrence, generally involving cutaneous and abdominal manifestations, especially during a 2-year period after the first outbreak. ${ }^{1-3}$

Some patients may progress with chronic kidney disease, characterized by glomerulonephritis, systemic arterial hypertension and compromised renal function. ${ }^{4,5}$

The objective of this study was to describe a group of patients treated for HSP at our service, putting emphasis on describing recurrent and chronic cases.

\section{Methods}

This study received prior approval from the Medical Ethics Committee at the Universidade Federal de São Paulo/Hospital São Paulo.

Medical records were reviewed retrospectively for patients who were first seen at our service between 1995 and 2004, presenting with HSP. Diagnosis was made based on American College of Rheumatology criteria. ${ }^{6}$ A minimum follow-up period of three months was one of the inclusion criteria. Cases were excluded if they had merely been referred for a specialist opinion, as were patients who ceased attending follow-up appointments for whatever reason, and those with comorbidities.

The demographic (age and sex), clinical (trigger factor, presence of purpura, arthritis, abdominal pains, gastrointestinal hemorrhage and renal involvement) and therapeutic characteristics (corticoids and prophylaxis with benzathine penicillin) of 55 patients were studied. At our service, we are currently prescribing benzathine penicillin every 21 days, for a period varying from six to 24 months, for patients with recurrent or chronic HSP, even in the absence of serological evidence of streptococcal infection, based on the possibility that the true the disease is triggered by Streptococcus, which is a common bacterium in our country. Also routine, is oral or intravenous corticoid in cases of moderate/severe gastrointestinal involvement, pulmonary hemorrhage, central nervous system involvement and orchiepididimitis.

When there is renal involvement, depending on the histological results of the kidney biopsy, it may become necessary to use immunosuppression, with azathioprine being the drug of choice at our service in the majority of cases.
Recurrence was defined as the presence of a fresh episode after a period of at least 3 months without signs or symptoms. Cases were defined as chronic when cutaneous, gastrointestinal and/or renal manifestations persisted for a continuous period of 12 months or more.

The following definitions were adopted for renal involvement: microscopic hematuria (> 10 erythrocytes/ field), macroscopic hematuria (> 100 erythrocytes/field), proteinuria (> $5 \mathrm{mg} / \mathrm{kg} / 24 \mathrm{~h}$ ) and systemic arterial hypertension ( $>$ 90th percentile for arterial systolic and diastolic pressure for age and stature).

Trigger factors were investigated by means of interviews with parents, and included the occurrence of infections (upper/lower airway infections or acute gastroenterocolitis), consumption of foods to which the patient is not accustomed and use of medicines or vaccinations during the 7 days prior to onset of clinical status.

Statistical analysis was performed using Student's t test for continuous variables and wither Fisher's or the chi-square test for categorical variables. Results were considered statistically significant where $p<0.05$.

\section{Results}

Demographic and clinical data on the 55 patients are presented in Table 1. Recurrence was observed in eight patients $(14.4 \%)$ and four $(7.2 \%)$ cases were chronic.

When we compared the subset of patients with monocyclic disease ( $n=43$ patients) with the subset of patients with recurrent or chronic cases ( $n=12)$, we observed that there was a significant difference between their mean ages (5.4 vs. 8.4 years, respectively; $p=0.011$ ).

Twenty-seven patients were given corticoids, and in 21 of these the indication was gastrointestinal involvement. There were no cases of severe complications, such as intestinal bleeding or perforation. Where it was necessary to give corticoid therapy intravenously, we used methylprednisolone at dosages of 1 to $2 \mathrm{mg} / \mathrm{kg} /$ day, split between two to four daily doses, always in association with intravenous ranitidine. The corticoid of choice for oral route was prednisolone or prednisone, also in association with oral ranitidine. Six patients were also given corticoids because of orchitis or severe cutaneous involvement.

All eight patients who relapsed exhibited cutaneous manifestation in their later attacks. Additionally, three patients presented arthritis; four abdominal pains; and seven had renal involvement: microscopic hematuria $(n=5)$, macroscopic hematuria $(n=1)$ and hematuria with proteinuria $(n=1)$.

Four patients progressed chronically (altered urinary sedimentation) and underwent renal biopsy. One 9-year-old 
girl with chronic cutaneous and renal involvement exhibited necrotizing mesangial proliferative diffuse glomerulitis with increasing fibrocells and mesangial IgA and C3 deposits. One 6-year-old boy with macroscopic hematuria was diagnosed with diffuse proliferative mesangial glomerulonephritis with IgA deposits. An 11-year-old girl who continues to suffer from hematuria and proteinuria exhibited mesangial IgA deposits. Finally, minimal lesions were observed in a 10-year-old girl with hematuria and proteinuria lasting 18 months.

Patients with recurrent purpura exhibited a greater frequency of renal involvement when compared with patients with monocyclic disease $(p=0.015)$. We also observed that arthritis present during the first attack correlated with chronic cases $(p=0.022)$.

\section{Discussion}

Monocyclic HSP is most often vasculitis observed in day-to-day pediatric practice. Nevertheless, around 20 to $30 \%$ of patients may exhibit relapse, especially during the 2 years following their first episode. ${ }^{2,3}$ In this study we observed recurrence and chronicity rates of 14.4 and $7.2 \%$, respectively.

There are descriptions of HSP affecting children of all ages, being more prevalent in schoolchildren. ${ }^{2,7}$ In Brazil, Silva et al. have observed peak incidence at 6 years of age, which is similar to the findings with our patient sample. Observed frequency was greater among girls (31 girls vs. 24 boys), in contrast with the literature, where a majority of studies report a discrete male predominance. ${ }^{1,3,7-9}$
Sano et al. ${ }^{10}$ assessed 134 HSP patients and observed that age of more than 4 years at disease onset was associated, among other factors, with renal involvement with worse prognosis. We did not observe any association between age and renal prognosis; however we did note a correlation with relapse rate, i.e. the mean age of patients with recurrent disease was greater than that of patients with monocyclic cases (8.4 and 5.4 years, respectively; $\mathrm{p}=0.011$ ).

It is to be recommended that possible trigger factors be investigated when dealing with HSP patients, since they may be responsible for recurrent cases. In a recent study, Gonzáles-Gay et al. ${ }^{1}$ described the presence of URI preceding $41 \%$ of HSP cases and observed a positive correlation between this factor and increased incidence of nephritis, although there was no effect on final disease prognosis. In our study, we detected a previous history of infection in approximately $50 \%$ of cases, including URI and acute gastroenterocolitis, with no correlation with recurrence, chronicity or prognosis. Although many studies have identified beta-hemolytic streptococcus as a trigger factor for $\mathrm{HSP}_{1}{ }^{1,3,8}$ there are no controlled studies that confirm the benefits of benzathine penicillin for prophylaxis against relapse. In our sample this conduct was indicated for eight relapsed patients with, all of whom responded well.

No statistically significant relationship was observed between the use of corticoids and recurrence, chronicity or prognosis.

Table 1 - Demographic and clinical characteristics of 55 patients with HSP, broken down into monocyclic or recurrent/chronic clinical course

\begin{tabular}{|c|c|c|c|}
\hline & \multirow[b]{2}{*}{ Total $(n=55)$} & \multicolumn{2}{|c|}{ Clinical course } \\
\hline & & Monocyclic $(n=43)$ & ${\text { Recurrent } / \text { chronic }^{+}(n=12)}$ \\
\hline Age (years)* & $6.0 \pm 2.5 \mathrm{SD}$ & $5.4 \pm 2.5 \mathrm{SD}$ & $8.4 \pm 2.4 \mathrm{SD}$ \\
\hline Sex (female/male) & $31 / 24(56 / 44 \%)$ & $26 / 17(60 / 40 \%)$ & $5 / 7(41 / 59 \%)$ \\
\hline History of infection & $29(52.7 \%)$ & $26(60.4 \%)$ & $3(25 \%)$ \\
\hline Purpura & $55(100 \%)$ & $43(100 \%)$ & $12(100 \%)$ \\
\hline Arthritis & $32(58.1 \%)$ & $29(67.4 \%)$ & $3(25 \%)$ \\
\hline Abdominal pains & $25(45.4 \%)$ & $21(48.8 \%)$ & $4(33.3 \%)$ \\
\hline Renal involvement & $9(16.3 \%)$ & $2(4.6 \%)$ & $7(58.3 \%)$ \\
\hline Microscopic hematuria & $7(12.7 \%)$ & $2(4.6 \%)$ & $5(41.6 \%)$ \\
\hline Macroscopic hematuria & $1(1.8 \%)$ & - & $1(8.3 \%)$ \\
\hline Proteinuria and hematuria & $1(1.8 \%)$ & - & $1(8.3 \%)$ \\
\hline
\end{tabular}

HSP = Henoch-Schönlein purpura; SD = standard deviation.

$* p=0.01$.
+ The subset of patients with recurrent/chronic clinical course comprised eight patients with recurrent disease and 4 patients with chronic cases. 
The main determinant of HSP outcome is renal involvement, affecting 10 to $50 \%$ of patients, generally during the first 3 months of the disease. ${ }^{3,9}$ The most common alterations are microscopic hematuria and mild proteinuria. Other less common findings are nephrotic syndrome and arterial hypertension, which may correlate with poor prognosis and chronic renal failure. ${ }^{2,8,10,11}$ In our sample, we observed microscopic hematuria in $12.7 \%$ of our patients, with a greater percentage among recurrent cases $(41.6 \%)$. Furthermore, in general we observed a significant correlation $(p=0.015)$ between renal involvement and the possibility of cutaneous relapse. Despite the anatomopathological findings in four of the patients we took biopsies from, none of them suffered kidney failure. It should be pointed out that this subset of patients should be followed-up over the long term, with periodical urinary sediment analyses and renal function tests (serum urea and creatinine and creatinine breakdown).

Silva et al. ${ }^{3}$ reported arthritis and/or arthralgia in $78 \%$ of cases, which is similar to our data (58.1\%). Although we observed a statistically significant relationship between arthritis and chronic cases ( $p=0.022$ ), we should interpret this finding with caution, since involvement of joints is common in HSP and no similar data are reported in the literature.

We suggest the standardization of investigations into possible trigger factors for chronic and recurrent cases: question parents and patients about possible infections suffered by the patient or day-to-day contacts during the 30 days prior to HSP onset and as the disease progresses, and also a detailed discussion of habitual and abnormal dietary items, including consumption of foods with large quantities or colorings and preservatives and of medicines such as vitamins, anti-inflammatories and antibiotics. Investigation can involve excluding potential triggers from the diet and performing tests for specific allergens, such as the radio-allergo-sorbent-test (RAST).

Our data reiterates the need for periodic follow-up of all children with HSP, especially in cases with renal involvement, such as, for example, those with persistent hematuria and proteinuria or arterial hypertension. Urinary sediments should be monitored, even in asymptomatic patients. Referral to a specialist (pediatric rheumatologist or nephrologist) is recommended in cases where presentation is atypical.

\section{References}

1. Gonzalez-Gay MA, Calvino MC, Vazquez-Lopez ME, GarciaPorrua C, Fernandez-Iglesias JL, Dierssen T, et al. Implications of upper respiratory tract infections and drugs in the clinical spectrum of Henoch-Schönlein purpura in children. Clin Exp Rheumatol. 2004;22:781-4.

2. Saulsbury FT. Henoch-Schönlein purpura. Curr Opin Rheumatol. 2001;13:35-40.

3. Silva CAA, Campos LMMA, Liphaus BL, Kiss MHB. Púrpura de Henoch-Schönlein na criança e no adolescente. Rev Bras Reumatol. 2000;40:128-36.

4. Ronkainen J, Ala-Houhala M, Huttunen NP, Jahnukainen T, Koskimies O, Ormala T, et al. Outcome of Henoch-Schönlein nephritis with nephrotic-range proteinuria. Clin Nephrol. 2003;60:80-4.

5. Stewart M, Savage JM, Bell B, McCord B. Long term renal prognosis of Henoch-Schönlein purpura in an unselected childhood population. Eur J Pediatr. 1988;147:113-5.

6. Mills JA, Michel BA, Bloch DA, Calabrese LH, Hunder GG, Arend WP, et al. The American College of Rheumatology 1990 criteria for the classification of Henoch-Schönlein purpura. Arthritis Rheum. 1990;33:1114-21.

7. Ting TV, Hashkes PJ. Update on childhood vasculitides. Curr Opin Rheumatol. 2004;16:560-5.

8. Ballinger S. Henoch-Schönlein purpura. Curr Opin Rheumatol. 2003;15:591-4.

9. Szer IS. Henoch-Schönlein purpura. Curr Opin Rheumatol. 1994;6:25-31.

10. Sano $H$, Izumida $M$, Shimizu $H$, Ogawa $Y$. Risk factors of renal involvement and significant proteinuria in Henoch-Schönlein purpura. Eur J Pediatr. 2002;161:196-201.

11. Kawasaki Y, Suzuki J, Sakai N, Nemoto K, Nozawa R, Suzuki S, et al. Clinical and pathological features of children with HenochSchönlein purpura nephritis: risk factors associated with poor prognosis. Clin Nephrol. 2003;60:153-60.

Correspondence:

Claudio A. Len

Rua dos Otonis 725, Vila Clementino

CEP 04025-002 - São Paulo, SP - Brazil

Tel./Fax: +55 (11) 5579.1590

E-mail: len.cl@terra.com.br 